\title{
Exploring the Relationship between the Platelet Indices and Psychosocial Morbidity in Elderly Patients at a Rural Medical College Hospital
}

\author{
Sunil Kumar*, Abhijit Agrawal, Jahanvi Bhagwati and Sachin Agrawal \\ Jawahar Lal Nehru Medical College, Datta Meghe Institute of Medical Sciences (DU), Wardha, Maharashtra, India \\ *Corresponding author: ISunil Kumar, MD, PGDGM (Geriatric medicine), Professor and Head, Department of Medicine, Jawahar Lal Nehru Medical \\ College, Meghe Institute of Medical Sciences (DU), Wardha, Maharashtra, India
}

Submission: 眥 March 05, 2018; Published: 轁 May 07, 2018

\begin{abstract}
Background: The platelet indices like mean platelet volume (MPV), platelet distribution width (PDW), and plateletcrit (PCT) are a laboratory marker associated with platelet function and activity. We have tried to correlate and compare all these indices in elderly patients as far as psychosocial morbidity like cognition, frailty and depression are concerned.

Methods: This was a prospective observational study and it comprised 1000 consecutive elderly patients admitted for routine check-up in medicine department. Blood samples were taken to measure platelet indices like MPV, PDW and PCT. Cognition, frailty and depression were assessed by the Mini mental state examination, frail non disabled questionnaire and Geriatric Depression scale respectively.

Result: Patients with normal and high platelet count had a depression score of more than 5. [(p=0.0001, $r=0.705) \chi 2=811.21, p=0.0031, S]$. In the group of low platelet count, $50.58 \%$ patients were robust, $49.42 \%$ patients were frail $(\mathrm{r}=0.792, \mathrm{p}=0.0001)$. Among normal platelet count group, $92.75 \%$ were frail $(\mathrm{r}=0.337, \mathrm{p}=0.0001)$. In high platelet count group, $13.40 \%$ were robust, $71.30 \%$ frail and $15.30 \%$ were disable $(\mathrm{r}=0.781, \chi 2=652.91$; $\mathrm{p}<0.0001, \mathrm{~S})$. In high platelet count group $81.01 \%$ had MMSE score $\leq 23,(\mathrm{r}=-0.440, \chi 2=754.72, \mathrm{p}=0.0001, \mathrm{~S}) .79 .25 \%$ patients having high PCT had MMSE score $\leq 23,(\mathrm{r}=-0.528, \chi 2=737.28, \mathrm{p}=0.0001, \mathrm{~S})$.
\end{abstract}

Conclusion: Platelet indices could be considered meaningful laboratory markers for the risk of psychosocial morbidity in elderly people.

Keywords: Elderly; Psychosocial; Morbidity; Platelet indices

\section{Introduction}

Ageing is a normal, progressive and irreversible process which affects every individual, family, community and society. Government of India under National Policy on Older Persons has defined 'senior citizen' or 'elderly' as a person who is of age 60 years or above in 1999. In our country total population of elderly is 10 crore, forming $10 \%$ of total population and it is estimated to reach up to 15 crore by 2020 [1]. Among them at least $50 \%$ have chronic diseases thus posing a greater responsibility on the health services where there is a greater strain on available health infrastructure and financial constraints [2]. Aging is a process of deterioration in the functional capacity of an individual that results from structural changes, with advancement of age leading to an increased risk of functional dependence, hospitalization, and mortality [3-5].

Therefore, many recent studies have investigated the usefulness of various parameters of automated blood cell counters as biomarkers for adverse clinical outcomes in the elderly. Among them is total leucocytes and some subpopulations of leucocytes which is associated with frailty, red blood cell distribution width seems to be a good indicator of morbidity, and mean platelet volume as markers for cardiovascular disease and its risk factors like hypertension and diabetes [6-12]. The platelet indices like mean platelet volume (MPV), platelet distribution width (PDW), and plateletcrit (PCT) values exhibited significant variability. There is evidence that Depression is associated with platelet activation, it also became apparent that the selective serotonin reuptake inhibitor (SSRIs) in general and sertraline in particular affect platelet function. SSRIs block reuptake of serotonin not only in nerve cells but also in platelets as well $[13,14]$.

Common psychosocial problems include impaired memory and intelligence, anxiety, depression, frailty, dependency and dissatisfaction with family members, earning and occupation. There is limited knowledge of the usefulness of platelet indices as markers for psychosocial morbidity like depression, frailty, and cognition in the elderly. Research is needed to identify risk 
factors at a stage of age-related decline that would be amenable to preventive interventions. To address this issue, we have tried to analyse the association of the following platelet indices as Platelet count (PLT), platelet distribution width (PDW), MPV, and platelet crit with psychosocial morbidity as a potential biomarkers.

\section{Material and Method}

This prospective observational study was conducted in rural medical hospital at Wardha city, the state of Maharashtra from August 2016 to July 2017. All consecutive patients above the age of 60 years admitted in our hospital were enrolled for the study, after taking approval from the institutional ethics committee. In our hospital there is Adhar scheme which provides free of cost screening and treatment for the patients having average daily admission of 15 to 20 patients, being most of them elderly. We have enrolled 1200 elderly patients under this scheme who has come for routine checkup. Patients having malignant disease, infection, h/o blood transfusion, myeloproliferative disorder, h/o recent major surgery, h/o intake of anti-platelet drugs, h/o chemotherapy/radiotherapy and critically ill were excluded from the study. Total 200 patients were excluded from the study and 1000 were enrolled (Figure 1).

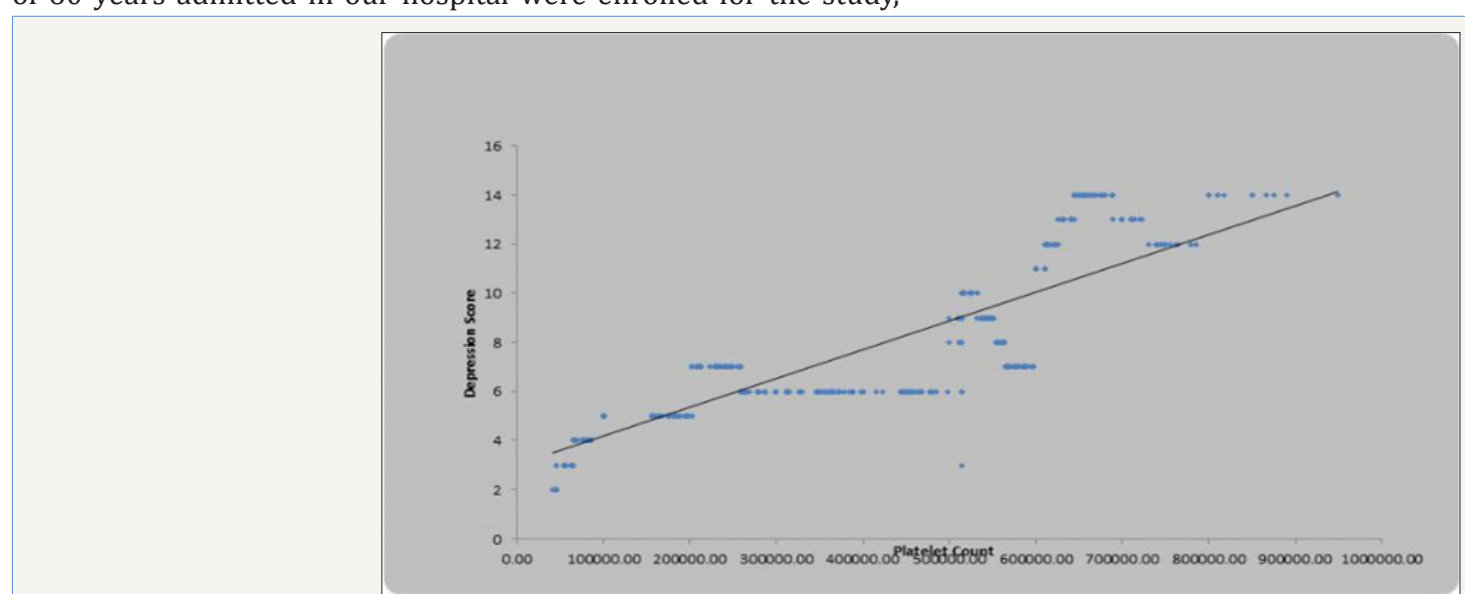

Figure 1: Scatter diagram showing linear correlation between platelet count and depression score.

Review was done regarding subject's medical and pharmacological history, a physical examination, and a physical activity questionnaire. The disease status of the patient was based on the explicit diagnosis in the patient's medical history. Patient's physical activity and daily life were evaluated on the basis of their frailty index. The diagnosis of dementia and cognitive impairment was based on MMSE and the criteria of the diagnostic and statistical manual of the mental disorder. Each participant's relatives were provided with information about the purpose and objective of the study and signed informed consent. The Geriatric Depression scale was used to assess the probable depression in our study group. A score of $\geq 5$ on geriatric depression scale was suggestive of probable depression.

The frailty score (FIND-frail non disabled questionnaire) was used for diagnosing the frailty. A patient with score $a+b=1$ was labelled "ROBUST", a patient with score $c+d+e=1$ was labelled "FRAIL" and patient with a score $\mathrm{a}+\mathrm{b}+\mathrm{c}+\mathrm{d}+\mathrm{e}=0$ was labelled as "DISABLED". MMSE (mini mental state examination) questionnaire was used for diagnosing cognitive impairment. A score of $\leq 23$ was suggestive of cognitive impairment.

\section{Blood collection}

Blood samples were obtained by venupuncture for biochemical analysis. Following platelet indices had been measured using automated hematology analyzer in the central laboratory of the hospital-platelet count, mean platelet volume and platelet distribution width and platelet crit.
Definitions:

A. Platelet count: Total number of platelets (1.5lac/cu mm to 5.0lac/cu mm).

B. Plateletcrit: It is a measure of total platelet mass (0.150 to $0.500 \%)$.

C. Mean platelet volume: It is the average size of platelets (6.0 to $11.0 /$ cu micrometer).

D. Platelet distribution width: It is the variability in the size of the smallest and the largest platelets (11.0 to $18.0 \%$ ).

\section{Statistical analysis}

Statistical analysis was done by using descriptive and inferential statistics using Chisquare test, Pearsons' correlation coefficient and software used in the analysis were SPSS 20.0 version, Graph Pad Prism 6.0 version and $p<0.05$ is considered as level of significance.

\section{Result and Observation}

In this study we included 1000 patients of whom 601(60.1\%) were males and 399(39.9\%) were females, mean age was $66.02 \pm 5.76$ years. The mean platelet count was $303210 \pm 21296 \mathrm{lac} /$ cu $\mathrm{mm}$, the mean platelet crit $0.29 \pm 0.17 \%$, mean platelet volume was $9.25 \pm 5.70$ per $\mu \mathrm{m}^{3}$, and the mean platelet distribution width $14.18 \pm 5.63 \%$. There were 165 males who were chronic smokers and 107 males who were chronic alcoholics.

In our study of 1000 patients, 259 patients had platelet count less than 1.5lac/cu mm of which 222(85.71\%) patients had a 
depression score of less than 5 and $37(14.29 \%)$ patients had a depression score of more than $5(\mathrm{p}=0.0001, \mathrm{r}=0.945) .483$ patients with normal platelet count had a depression score more than 5 , ( $p=0.0001, r=0.202)$. Out of 258 patients 257(99.61\%) patients

Table 1: Correlation of platelet count with depression score. had a depression score of more than 5 , which was statistically significant $[(p=0.0001, r=0.705) \times 2=811.21, p=0.0031, S]$. The correlation of platelet count with Depression score is shown in Figure 1 and Table 1.

\begin{tabular}{|c|c|c|c|c|c|}
\hline \multirow{2}{*}{ Platelet Count } & \multirow{2}{*}{$\mathbf{n}$} & \multicolumn{2}{|c|}{ Depression Score } & \multirow{2}{*}{ r-value } & \multirow{2}{*}{ p-value } \\
\cline { 2 - 5 } & & $<$ & $37(14.29 \%)$ & 0.945 & $0.0001, \mathrm{~S}$ \\
\hline Low(<1.5 lakhs) & 259 & $222(85.71 \%)$ & $483(100 \%)$ & 0.202 & $0.0001, \mathrm{~S}$ \\
\hline Normal(1.5-5 lakhs) & 483 & $0(0 \%)$ & $257(99.61 \%)$ & 0.705 & $0.0001, \mathrm{~S}$ \\
\hline High(>5 lakhs) & 258 & $1(0.39 \%)$ & $777(77.70 \%)$ & 0.874 & $0.0001, \mathrm{~S}$ \\
\hline Total & 1000 & $223(22.3 \%)$ & \multicolumn{2}{|c|}{$811.21, \mathrm{p}=0.0031, \mathrm{~S}$} \\
\hline & & $\chi 2$-value & \multicolumn{2}{|c|}{$0.001(95 \% \mathrm{CI}=0.00008-0.021)$} \\
\hline
\end{tabular}

In lower platelet count group, 131(50.58\%) patients were in and 32(6.63\%) were disable, $(r=0.337, p=0.0001)$. Among higher robust category according to frailty score , 128(49.42\%) frail and $0 \%$ were disable, having positive correlation between platelet count and frailty score $(\mathrm{r}=0.792$ and $\mathrm{p}=0.0001)$. In patients having normal platelet $3(0.62 \%)$ were robust, $448(92.75 \%)$ were frail platelet count group, 134(13.40\%) patients were robust, 713 $(71.30 \%)$ were frail and $153(15.30 \%)$ were disable, ( $r=0.781$, $\chi 2=652.91 ; p<0.0001, S)$. The Correlation of platelet count with Frailty score is shown in Figure 2 and Table 2.

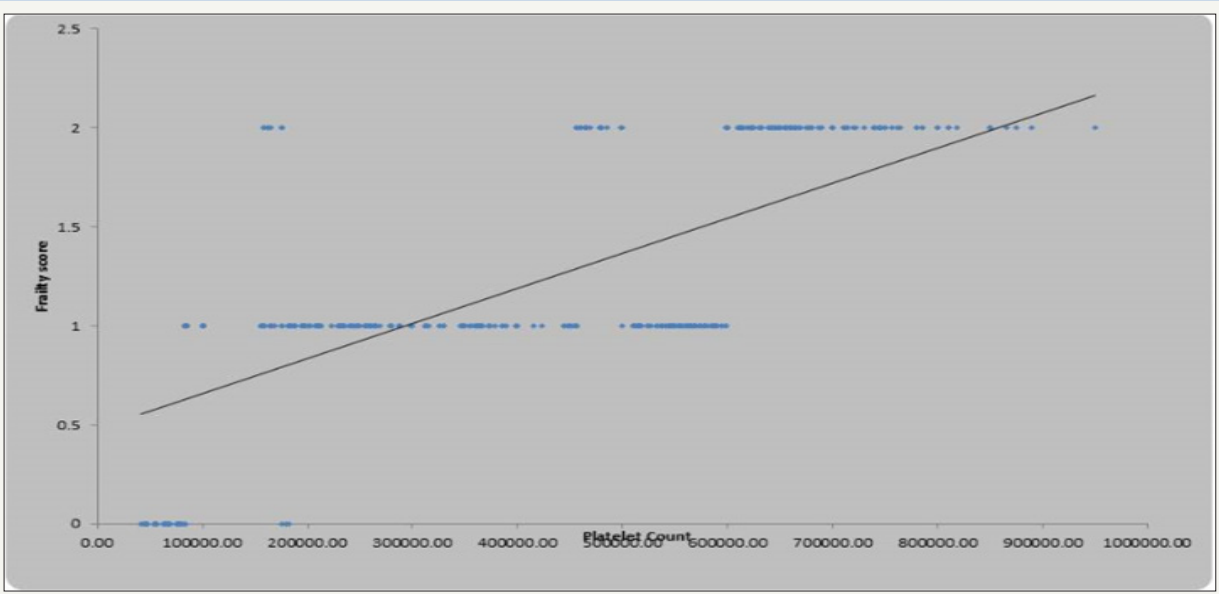

Figure 2: Scatter diagram showing linear correlation between platelet count and frailty score.

Table 2: Correlation of platelet count with Frailty score.

\begin{tabular}{|c|c|c|c|c|c|c|}
\hline \multirow{2}{*}{ Platelet Count } & \multirow{2}{*}{$\mathbf{n}$} & \multicolumn{3}{|c|}{ Frailty Score } & \multirow{2}{*}{ r-value } & p-value \\
\cline { 3 - 6 } & 259 & Robust & Frail & Disable & 0.792 & $0.0001, \mathrm{~S}$ \\
\hline Low(<1.5 lakhs) & 483 & $3(0.62 \%)$ & $448(92.75 \%)$ & $32(6.63 \%)$ & 0.337 & $0.0001, \mathrm{~S}$ \\
\hline $\begin{array}{c}\text { Normal(1.5-5 } \\
\text { lakhs) }\end{array}$ & 258 & $0(0 \%)$ & $137(53.10 \%)$ & $121(46.90 \%)$ & 0.781 & $0.0001, \mathrm{~S}$ \\
\hline High(>5 lakhs) & 1000 & $134(13.40 \%)$ & $713(71.30 \%)$ & $153(15.30 \%)$ & 0.705 & $0.0001, \mathrm{~S}$ \\
\hline Total & \multicolumn{7}{|c|}{$\chi 2$-value } & & $652.91, \mathrm{p}=0.0001, \mathrm{~S}$ \\
\hline
\end{tabular}

Table 3: Correlation of platelet count with MMSE score.

\begin{tabular}{|c|c|c|c|c|c|}
\hline \multirow{2}{*}{ Platelet Count } & \multirow{2}{*}{$\mathbf{n}$} & \multicolumn{2}{|c|}{ MMSE Score } & \multirow{2}{*}{ r-value } & \multirow{2}{*}{ p-value } \\
\cline { 2 - 5 } & & $\mathbf{5 2 3}$ & $\mathbf{2 3}$ & 0.705 & $0.0001, \mathrm{~S}$ \\
\hline Low(<1.5 lakhs) & 259 & $0(0 \%)$ & $259(100 \%)$ & -0.133 & $0.003, \mathrm{~S}$ \\
\hline Normal(1.5-5 lakhs) & 483 & $1(0.21 \%)$ & $482(99.79 \%)$ & & \\
\hline
\end{tabular}




\begin{tabular}{|c|c|c|c|c|c|}
\hline $\operatorname{High}(>5$ lakhs $)$ & 258 & $209(81.01 \%)$ & $49(18.99 \%)$ & -0.509 & $0.0001, \mathrm{~S}$ \\
\hline \multirow[t]{2}{*}{ Total } & 1000 & $210(21 \%)$ & $790(79 \%)$ & -0.44 & $0.0001, \mathrm{~S}$ \\
\hline & \multicolumn{3}{|c|}{$\chi 2$-value } & \multicolumn{2}{|c|}{$754.72, p=0.0001 \mathrm{~S}$} \\
\hline Odd's Ratio & \multicolumn{5}{|c|}{$0.003(95 \% \mathrm{CI}=0.00042-0.021)$} \\
\hline
\end{tabular}

In our study in 259 patients with low platelet counts, none had MMSE score $\leq 23,(r=0.705, p=0.0001)$. Among patients with normal platelet count only $1(0.21 \%)$ patient had MMSE score $\leq 23$ and 482(99.79\%) patients had MMSE score $>23$, $(r=-0.133)$. In patients with high platelet count $209(81.01 \%)$ had MMSE score $\leq 23$ while 49(18.99\%) patients had MMSE score $>23$, ( $r=-0.509, p=0.0001)$. Altogether platelet count was negatively correlated with MMSE score $(r=-0.440)$ and it was statistically significant $(\chi 2=754.72$, $\mathrm{p}=0.0001 \mathrm{~S}$ ) (Table 3).
Among 294 patients with low platelet crit, 222(75.51\%) patients had depression score $<5$ and $72(24.49 \%)$ patients had depression score $\geq 5$, $(r=0.884, p=0.0001)$. All patients with normal platelet crit had depression score $\geq 5$, $(r=0.061, p=0.200)$. 265 patients had high platelet crit, of these $1(0.38 \%)$ patient had depression score $<5$ and 264(99.62\%) patients had depression score $\geq 5$, ( $r=0.696$, $\mathrm{p}=0.0001)$. Altogether platelet crit showed positive correlation with depression score $[(r=0.831, \chi 2=680.48 ; p<0.0001, S)]$, shown in Figure 3 and Table 4.

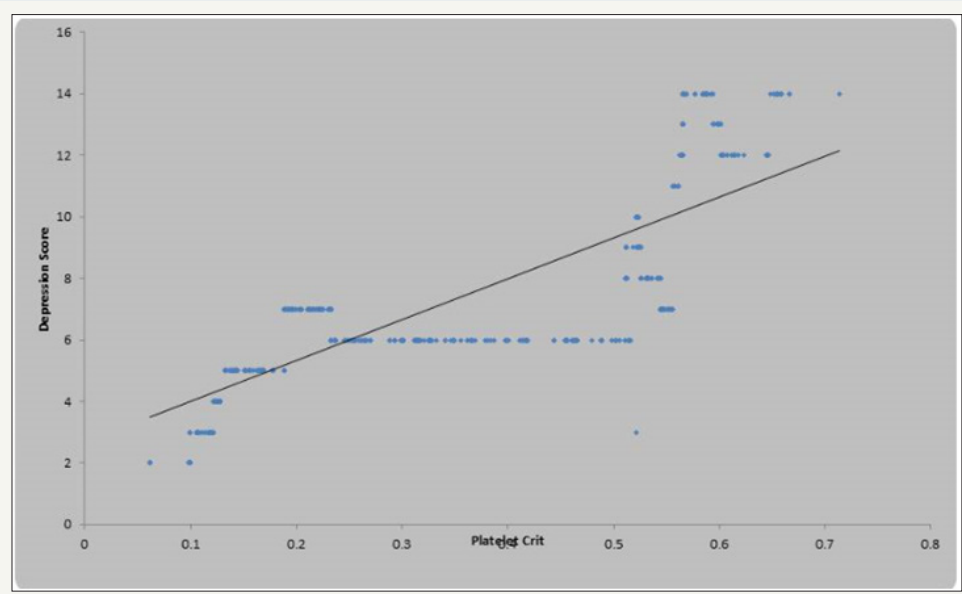

Figure 3: Scatter diagram showing linear correlation between platelet crit and depression score.

Table 4: Correlation of platelet crit with depression score.

\begin{tabular}{|c|c|c|c|c|c|}
\hline \multirow{2}{*}{ Platelet Crit } & \multirow{2}{*}{$\mathbf{n = 1 0 0 0}$} & \multicolumn{2}{|c|}{ Depression Score } & \multirow{2}{*}{ R-Value } & \multirow{2}{*}{ P-Value } \\
\cline { 2 - 5 } & & $\mathbf{<}$ & $72(24.49 \%)$ & 0.884 & $0.0001, \mathrm{~S}$ \\
\hline LOW(<0.150) & 294 & $222(75.51 \%)$ & $441(100 \%)$ & 0.061 & $0.2 .00, \mathrm{NS}$ \\
\hline NORMAL(0.150-0.500) & 441 & $0(0 \%)$ & $264(99.62 \%)$ & 0.696 & $0.0001, \mathrm{~S}$ \\
\hline HIGH(>0.500) & 265 & $1(0.38 \%)$ & $777(77.7 \%)$ & $0.0001, \mathrm{~S}$ \\
\hline TOTAL & 1000 & $223(22.30 \%)$ & & $680.48, \mathrm{p}=0.0001, \mathrm{~S}$ \\
\hline ODD'S RATIO & \multicolumn{7}{|c|}{$1.41(95 \% \mathrm{CI}=1.04-1.91)$} \\
\hline
\end{tabular}

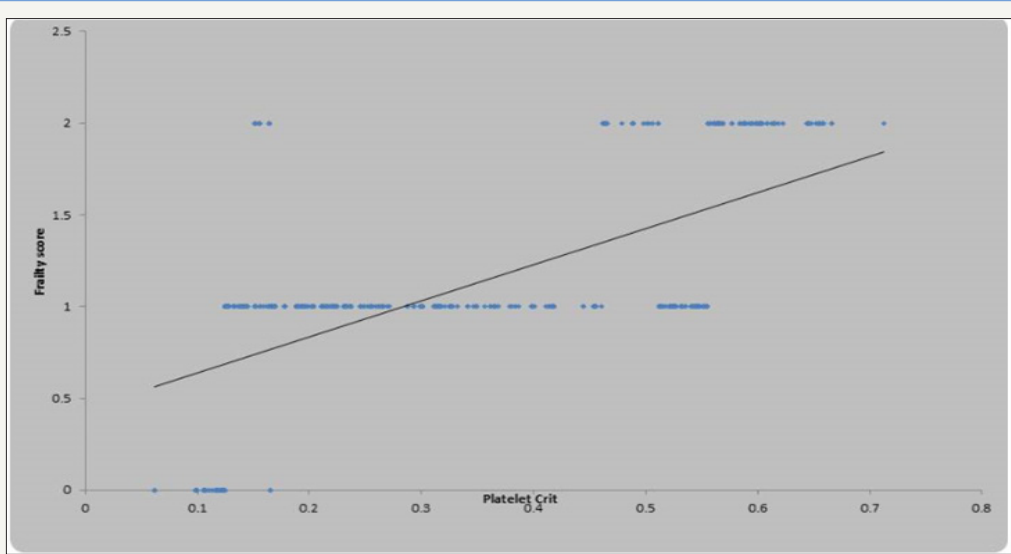

Figure 4: Scatter diagram showing linear correlation between platelet crit and frailty. 
Table 5: Correlation of platelet crit with frailty score.

\begin{tabular}{|c|c|c|c|c|c|c|}
\hline \multirow{2}{*}{ Platelet Crit } & \multirow{2}{*}{$\mathbf{n}$} & \multicolumn{3}{|c|}{ Frailty Score } & \multirow{2}{*}{ r-value } & \multirow{2}{*}{ p-value } \\
\hline & & Robust & Frail & Disable & & \\
\hline $\operatorname{Low}(<0.150)$ & 294 & $131(44.56 \%)$ & $163(55.44 \%)$ & $0(0 \%)$ & 0.651 & $0.0001, S$ \\
\hline $\begin{array}{c}\text { Normal }(0.150- \\
0.500)\end{array}$ & 441 & $3(0.68 \%)$ & $412(93.42 \%)$ & $26(5.90 \%)$ & 0.279 & $0.0001, S$ \\
\hline $\operatorname{High}(>0.500)$ & 265 & $0(0 \%)$ & $138(52.08 \%)$ & $127(47.92 \%)$ & 0.71 & $0.0001, \mathrm{~S}$ \\
\hline \multirow[t]{2}{*}{ Total } & 1000 & $134(13.40 \%)$ & $713(71.30 \%)$ & $153(15.30 \%)$ & 0.655 & $0.0001, \mathrm{~S}$ \\
\hline & \multicolumn{4}{|c|}{$\chi 2$-value } & \multicolumn{2}{|c|}{$610.95, \mathrm{p}=0.0001, \mathrm{~S}$} \\
\hline Odd's Ratio & \multicolumn{6}{|c|}{$6.68(95 \% \mathrm{CI}=4.27-10.46)$} \\
\hline
\end{tabular}

Out of 294 patients who had low platelet crit, 131(44.56\%) platelet crit with Frailty score is shown in Figure 4 and Table 5.

were robust, $163(55.44 \%)$ patients were frail and non were disable, $(\mathrm{r}=0.651, \mathrm{p}=0.0001)$. Patients with normal platelet crit (441), $3(0.68 \%)$ patients were robust, 412(93.42\%) patients were frail and $26(5.90 \%)$ patients were disable, $(\mathrm{r}=0.279, \mathrm{p}=0.0001)$. Patients with high platelet crit (265), non were robust, 138(52.08\%) patients were frail and $127(47.92 \%)$ patients were disable, $[(\mathrm{r}=0.710, \mathrm{p}=$ 0.0001) $(\chi 2=610.95 ; p<0.0001$ S-Significant $)]$. The Correlation of
In our study 294 patients had platelet crit below 0.150, of them none had MMSE score $\leq 23$, ( $\mathrm{r}=0.378,0.0001)$. In normal platelet crit group, none-had MMSE score $\leq 23$, ( $\mathrm{r}=0.052, \mathrm{p}=0.280) .265$ patients had high platelet crit ( $>0.150)$, among them $210(79.25 \%)$ had MMSE score $\leq 23$ and 55(20.75\%) had MMSE score $>23$, ( $r=-$ 0.528, $\mathrm{p}=0.0001),[\chi 2=737.28, \mathrm{p}=0.0001, \mathrm{~S}]$. The Correlation of platelet crit with MMSE score is shown in Figure 5 and Table 6.

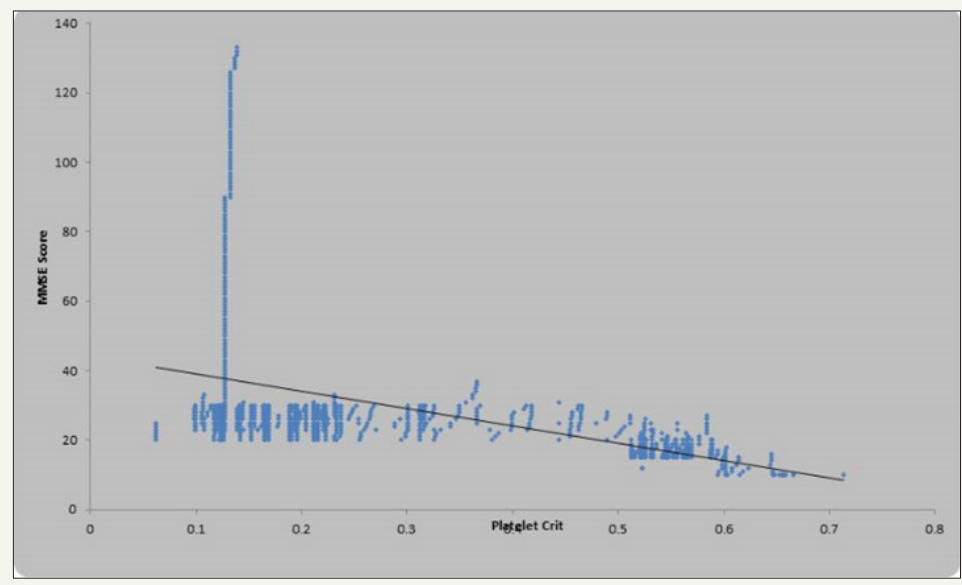

Figure 5: Scatter diagram showing linear correlation between platelet crit and MMSE score.

Table 6: Correlation of platelet crit with MMSE score.

\begin{tabular}{|c|c|c|c|c|c|}
\hline \multirow{2}{*}{ Platelet Crit } & \multirow{2}{*}{$\mathbf{n}$} & \multicolumn{2}{|c|}{ MMSE Score } & \multirow{2}{*}{ r-value } & \multirow{2}{*}{ p-value } \\
\hline & & $\leq 23$ & $>23$ & & \\
\hline $\operatorname{Low}(<0.150)$ & 294 & $0(0 \%)$ & $294(100 \%)$ & 0.378 & $0.0001, \mathrm{~S}$ \\
\hline Normal(0.150-0.500) & 441 & $0(0 \%)$ & $441(100 \%)$ & 0.052 & $0.280, \mathrm{NS}$ \\
\hline $\operatorname{High}(>0.500)$ & 265 & $210(79.25 \%)$ & $55(20.75 \%)$ & -0.528 & $0.0001, S$ \\
\hline \multirow[t]{2}{*}{ Total } & 1000 & $210(21 \%)$ & $790(79 \%)$ & -0.428 & $0.0001, S$ \\
\hline & \multicolumn{3}{|c|}{$\chi 2$-value } & \multicolumn{2}{|c|}{$737.28, p=0.0001, S$} \\
\hline Odd's Ratio & \multicolumn{5}{|c|}{$0.0018(95 \%$ CI $=0.00011-0.030)$} \\
\hline
\end{tabular}

In 259 patients with PDW <11\%, 222(85.71\%) had depression score $<5$ and $37(14.29 \%)$ had depression score $\geq 5$, ( $r=0.945$, $\mathrm{p}=0.0001$ ). 481 patients had normal PDW (11 to 18\%), of them all had depression score $\geq 5,(r=0.219, p=0.0001)$. In high PDW (18\%) group, out of $2601(0.38 \%)$ patient had depression score
$<5$ and 259(99.62\%) patients had depression score $\geq 5$, [( $\mathrm{r}=0.743$, $\mathrm{p}=0.0001)\left(\chi^{2}=811.21 ; \mathrm{p}<0.0001 \mathrm{~S}\right]$. The Correlation of platelet distribution width with Depression score is shown in Figure 6 and Table 7. 


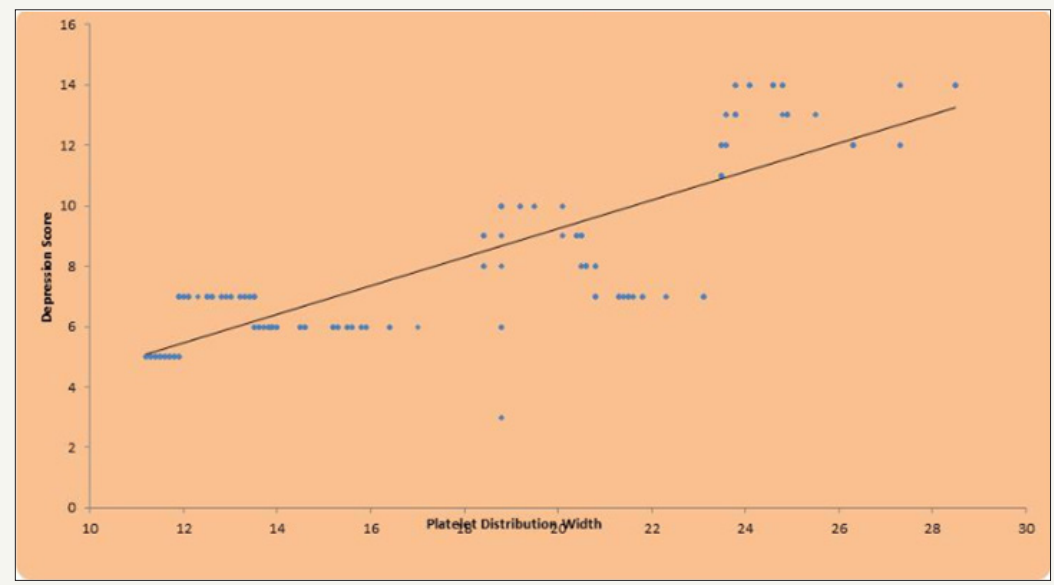

Figure 6: Scatter diagram showing linear correlation between platelet distribution width and depression score.

Table 7: Correlation of platelet distribution width with depression score.

\begin{tabular}{|c|c|c|c|c|c|}
\hline \multirow{2}{*}{$\begin{array}{c}\text { Platelet Distribution } \\
\text { Width }\end{array}$} & \multirow{2}{*}{$\mathbf{n}$} & \multicolumn{2}{|c|}{ Depression Score } & \multirow{2}{*}{ r-value } & \multirow{2}{*}{ p-value } \\
\hline & & $<5$ & $\geq 5$ & & \\
\hline Low $(<11 \%)$ & 259 & $222(85.71 \%)$ & $37(14.29 \%)$ & 0.945 & $0.0001, \mathrm{~S}$ \\
\hline Normal(11-18\%) & 481 & $0(0 \%)$ & $481(100 \%)$ & 0.219 & $0.0001, \mathrm{~S}$ \\
\hline $\operatorname{High}(>18 \%)$ & 260 & $1(0.38 \%)$ & $259(99.62 \%)$ & 0.743 & $0.0001, S$ \\
\hline \multirow[t]{2}{*}{ Total } & 1000 & $223(22.30 \%)$ & $777(77.70 \%)$ & 0.743 & $0.0001, S$ \\
\hline & \multicolumn{3}{|c|}{$\chi 2$-value } & \multicolumn{2}{|c|}{$811.21, \mathrm{p}=0.0001, \mathrm{~S}$} \\
\hline Odd's Ratio & \multicolumn{5}{|c|}{$0.0001(95 \% \mathrm{CI}=0.000085-0.022)$} \\
\hline
\end{tabular}

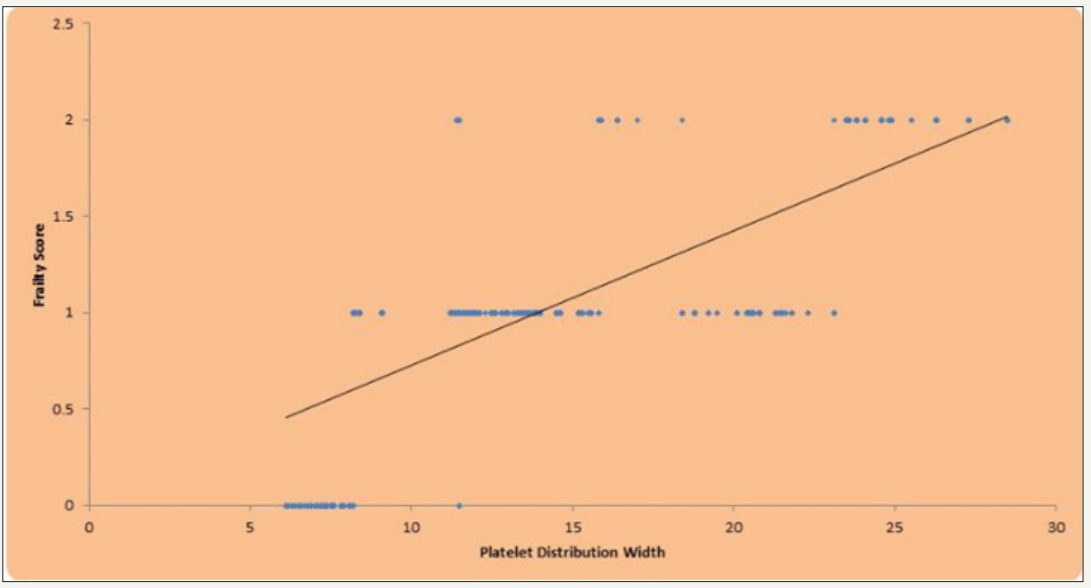

Figure 7: Scatter diagram showing linear correlation between platelet distribution width and frailty score.

Table 8: Correlation of platelet distribution width with frailty score.

\begin{tabular}{|c|c|c|c|c|c|c|}
\hline \multirow{2}{*}{$\begin{array}{c}\text { Platelet } \\
\text { Distribution } \\
\text { Width }\end{array}$} & \multirow[b]{2}{*}{$\mathbf{n}$} & \multicolumn{3}{|c|}{ Frailty Score } & \multirow[b]{2}{*}{ r-value } & \multirow[b]{2}{*}{ p-value } \\
\hline & & Robust & Frail & Disable & & \\
\hline Low $(<11 \%)$ & 259 & $131(50.58 \%)$ & $128(49.42 \%)$ & $0(0 \%)$ & 0.78 & $0.0001, \mathrm{~S}$ \\
\hline Normal(11-18\%) & 481 & $3(0.62 \%)$ & $447(92.93 \%)$ & $31(6.44 \%)$ & 0.283 & $0.0001, \mathrm{~S}$ \\
\hline $\operatorname{High}(>18 \%)$ & 260 & $0(0 \%)$ & $138(53.08 \%)$ & $122(46.92 \%)$ & 0.849 & $0.0001, \mathrm{~S}$ \\
\hline \multirow[t]{2}{*}{ Total } & 1000 & $134(13.40 \%)$ & $713(71.30 \%)$ & $153(15.30 \%)$ & 0.733 & $0.0001, S$ \\
\hline & \multicolumn{4}{|c|}{$\chi^{2 \text {-value }}$} & \multicolumn{2}{|c|}{$655.88, \mathrm{p}=0.0001, \mathrm{~S}$} \\
\hline Odd's Ratio & \multicolumn{6}{|c|}{$0.014(95 \% \mathrm{CI}=0.004-0.045)$} \\
\hline
\end{tabular}


According to frailty score $131(50.58 \%)$ were robust, had high PDW( $>18 \%)$, non among them were robust, 138(53.08\%) 128(49.42\%) were frail and none were disable in low PDW $(<11 \%)$ group, $(\mathrm{r}=0.780, \mathrm{p}=0.0001)$. Among 481 patients with normal PDW (11 to $18 \%), 3(0.62 \%)$ patients were robust, $447(92.93 \%)$ were frail and $31(6.44 \%)$ were disable, $(r=0.283, p=0.0001) .260$ patients patients were frail and $122(46.92 \%)$ patients were disable, $(r=0.849, p=0.0001),[(\times 2=655.88 ; p<0.0001 S]$. The Correlation of platelet distribution width with Frailty score is shown in Figure 7 and Table 8.

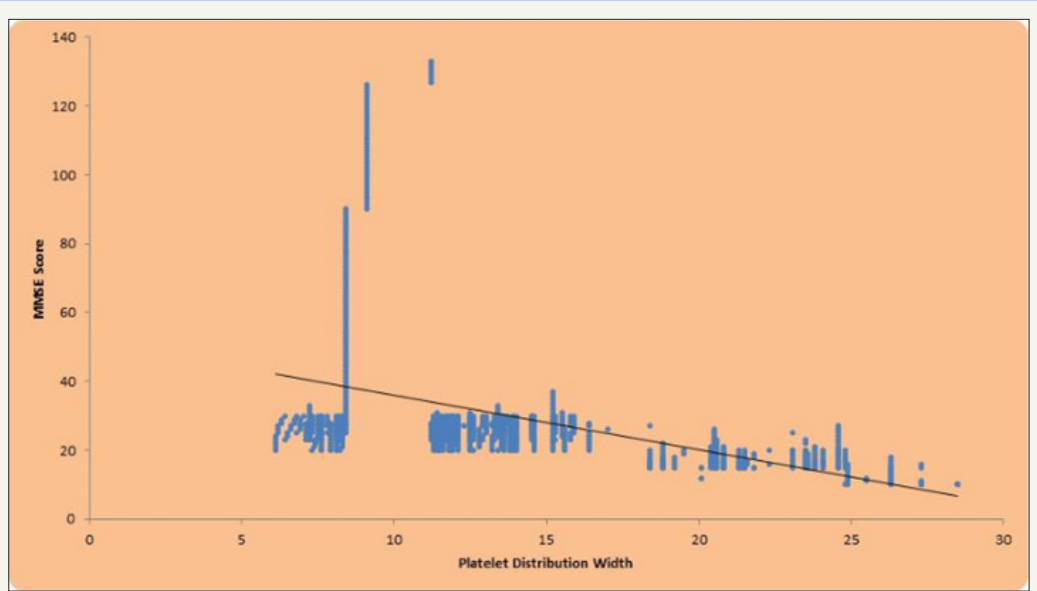

Figure 8: Scatter diagram showing linear correlation between platelet distribution width and MMSE score.

Table 9: Correlation of platelet distribution width with MMSE score.

\begin{tabular}{|c|c|c|c|c|c|}
\hline \multirow{2}{*}{$\begin{array}{c}\text { Platelet Distribution } \\
\text { Width }\end{array}$} & \multirow{2}{*}{$\mathbf{n}$} & \multicolumn{2}{|c|}{ MMSE Score } & \multirow{2}{*}{ r-value } & \multirow{2}{*}{ p-value } \\
\cline { 2 - 5 } & & $\mathbf{2 3}$ & $259(100 \%)$ & 0.72 & $0.0001, \mathrm{~S}$ \\
\hline Low $(<11 \%)$ & 259 & $0(0 \%)$ & $481(100 \%)$ & -0.139 & $0.002, \mathrm{~S}$ \\
\hline Normal(11-18\%) & 481 & $0(0 \%)$ & $50(19.23 \%)$ & -0.421 & $0.0001, \mathrm{~S}$ \\
\hline High(>18\%) & 260 & $210(80.77 \%)$ & $790(79 \%)$ & -0.428 & $0.0001, \mathrm{~S}$ \\
\hline Total & 1000 & $210(21 \%)$ & \multicolumn{2}{|c|}{$756.57, \mathrm{p}=0.0001, \mathrm{~S}$} \\
\hline
\end{tabular}

In low PDW group $(<11 \%)$ all had MMSE score $>23$, $(\mathrm{r}=0.720$, patients had MMSE score $>23$, $(\mathrm{r}=-0.421, \mathrm{P}=0.0001)$. Altogether p=0.0001). 481 patients with normal PDW (11 to $18 \%$ ), all had $210(21 \%)$ patients showed MMSE score $\leq 23$ and $790(79 \%)$ patient MMSE score $>23$, $(r=-0.139, p=0.002)$. In high PDW group (260), had MMSE score $>23,[(r=-0.428, \chi 2=756.57 ; p=0.0001)]$ Figure 8 $210(80.77 \%)$ patient had MMSE score $\leq 23$ and 50(19.23\%) and Table 9.

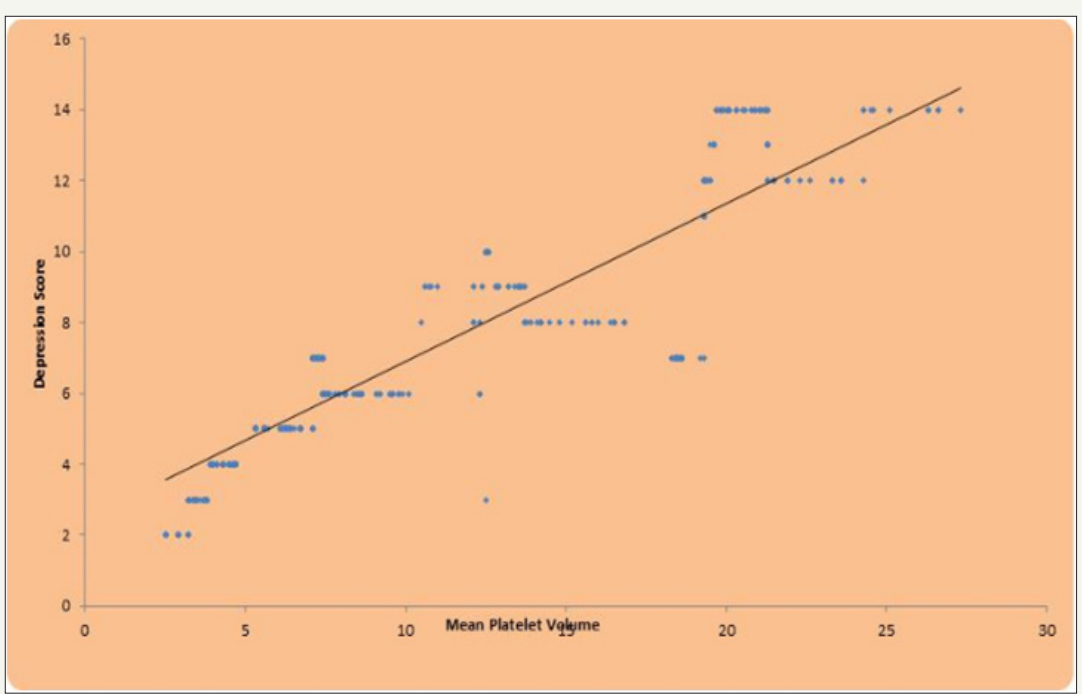

Figure 9: Scatter diagram showing linear correlation between mean platelet volume and Depression score. 
Table 10: Correlation of mean platelet volume width with depression score.

\begin{tabular}{|c|c|c|c|c|c|}
\hline \multirow{2}{*}{ Mean platelet volume } & \multirow{2}{*}{$\mathbf{n}$} & \multicolumn{2}{|c|}{ Depression Score } & \multirow{2}{*}{ r-value } & \multirow{2}{*}{ p-value } \\
\hline & & $<5$ & $\geq 5$ & & \\
\hline $\begin{array}{l}\text { Low }(<6.0 / \mathrm{cu} \\
\text { micrometer })\end{array}$ & 290 & $222(76.55 \%)$ & $68(23.45 \%)$ & 0.945 & $0.0001, \mathrm{~S}$ \\
\hline $\begin{array}{l}\text { Normal(6-11 cu } \\
\text { micrometer) }\end{array}$ & 456 & $0(0 \%)$ & $456(100 \%)$ & 0.391 & $0.0001, \mathrm{~S}$ \\
\hline $\begin{array}{l}\mathrm{High}(>11 \mathrm{cu} \\
\text { micrometer) }\end{array}$ & 254 & $1(0.39 \%)$ & $253(99.61 \%)$ & 0.605 & $0.0001, \mathrm{~S}$ \\
\hline \multirow[t]{2}{*}{ Total } & 1000 & $223(22.3 \%)$ & 777(77.7\%) & 0.889 & $0.0001, \mathrm{~S}$ \\
\hline & \multicolumn{3}{|c|}{$\chi 2$-value } & \multicolumn{2}{|c|}{$693.82, \mathrm{p}=0.002, \mathrm{~S}$} \\
\hline Odd's Ratio & \multicolumn{5}{|c|}{$0.0001(95 \%$ CI $=0.00009-0.025)$} \\
\hline
\end{tabular}

Out of 290 patients with low MPV $(<6.0 / \mathrm{cu} \mu \mathrm{m}), 222(76.55 \%)$ had depression score $<5$ and $68(23.45 \%)$ patients had depression score $\geq 5$, $(r=0.945, p=0.0001)$. All 456 patients with MPV (6.0 to $11.0 / \mathrm{cu} \mu \mathrm{m})$ had depression score $\geq 5$, ( $\mathrm{r}=0.391, \mathrm{p}=0.0001) .254$ patients had high MPV (>11/cu $\mu \mathrm{m})$, among them 253(99.61\%) patients had depression score $\geq 5$, $(r=0.605, p=0.0001)$. Out of 1000 patients $223(22.3 \%)$ patients had depression score $<5$ and $777(77.7 \%)$ patients had depression score $\geq 5$, [ $r=0.889, p=0.002$, $(\chi 2=693.82 ; p<0.0001, S]$. The correlation of mean platelet volume with depression score is shown in Figure 9 and Table 10.
In 290 patients with low MPV (<6.0/cu $\mu \mathrm{m}), 131(45.17 \%)$ were robust, $159(54.83 \%)$ were frail and none were disable, $(\mathrm{r}=$ $0.829,0.0001$ ). Among 456 patients with normal MPV ( 6 to $11 / \mathrm{cu}$ $\mu \mathrm{m}), 3(0.66 \%)$ were robust, $421(92.32 \%)$ were frail and $32(7.02 \%)$ were disable, $(\mathrm{r}=0.336, \mathrm{p}=0.0001)$. Among 254 patients having high MPV (>11/cu $\mu \mathrm{m})$, none were robust, $133(52.36 \%)$ were frail and $121(47.64 \%)$ were disable, $(r=0.769, p=0.0001)$. Of 1000 patients $134(13.40 \%)$ were robust, $712(71.30 \%)$ frail and $153(15.30 \%)$ were disable, $[(\mathrm{r}=0.705, \mathrm{p}=0.0001)(\chi 2=598.26 ; \mathrm{p}<0.0001 \mathrm{~S}]$ (Figure 10) (Table 11).

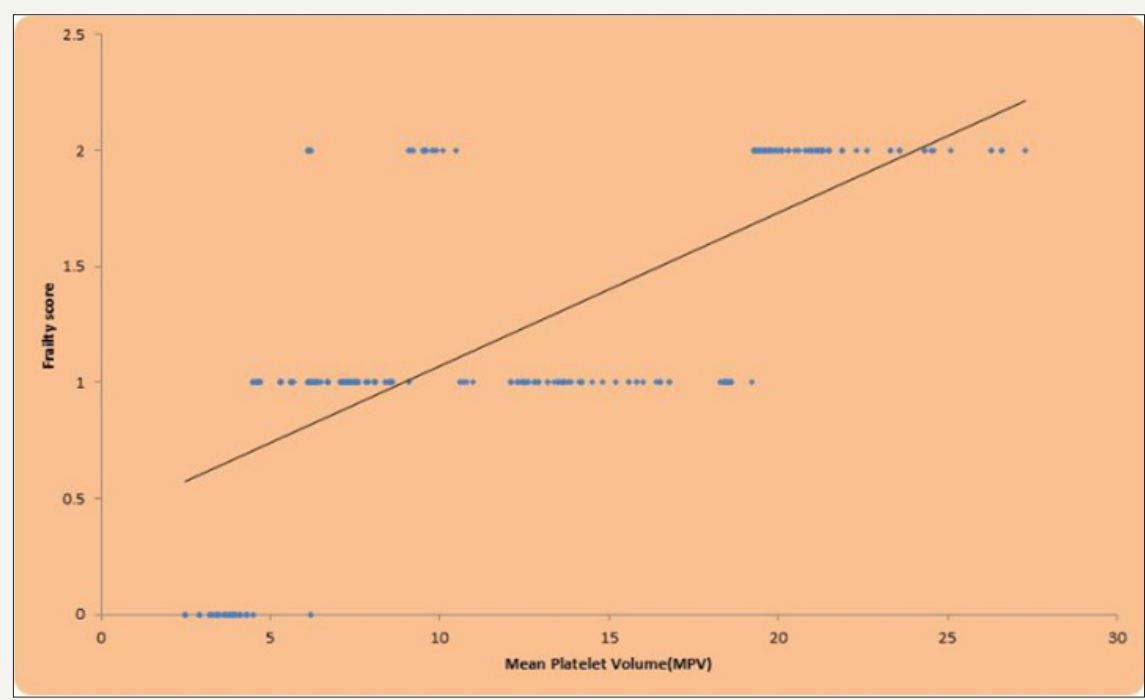

Figure 10: Scatter diagram showing linear correlation between mean platelet volume and frailty score.

Table 11: Correlation of mean platelet volume width with frailty score.

\begin{tabular}{|c|c|c|c|c|c|c|}
\hline \multirow{2}{*}{$\begin{array}{c}\text { Mean platelet } \\
\text { volume }\end{array}$} & \multirow{2}{*}{$\mathbf{n}$} & \multicolumn{3}{|c|}{ Frailty Score } & \multirow{2}{*}{ r-value } & p-value \\
\cline { 2 - 6 } $\begin{array}{c}\text { Low(<6.0/cu } \\
\text { micrometer) }\end{array}$ & 290 & $131(45.17 \%)$ & $159(54.83 \%)$ & $0(0 \%)$ & 0.829 & $0.0001, \mathrm{~S}$ \\
\hline $\begin{array}{c}\text { Normal (6-11 cu } \\
\text { micrometer) }\end{array}$ & 456 & $3(0.66 \%)$ & $421(92.32 \%)$ & $32(7.02 \%)$ & 0.336 & $0.0001, \mathrm{~S}$ \\
\hline $\begin{array}{c}\text { High(>11 cu } \\
\text { micrometer) }\end{array}$ & 254 & $0(0 \%)$ & $133(52.36 \%)$ & $121(47.64 \%)$ & 0.769 & $0.0001, \mathrm{~S}$ \\
\hline Total & 1000 & $134(13.40 \%)$ & $713(71.30 \%)$ & $153(15.30 \%)$ & 0.705 & $0.0001, \mathrm{~S}$ \\
\hline \multicolumn{7}{|c|}{$\chi 2$-value } \\
\hline Odd's Ratio & \multicolumn{7}{|c|}{$0.02(95 \% \mathrm{Cl}=0.006-0.066)$} & $598.26, \mathrm{p}=0.0001, \mathrm{~S}$ \\
\hline
\end{tabular}


In all patients (290) with low MPV $(<6.0 / \mathrm{cu} \mu \mathrm{m})$, MMSE score was $>23$, ( $r=0.568, p=0.0001)$. 451(98.90\%) patients with normal MPV had MMSE score $>23$, $(r=-0.105, p=0.025)$. Among 254 patients with high MPV, 205(80.71\%) had MMSE score $\leq 23$ and $49(19.29 \%)$ had MMSE score $>23$, $(r=-0.418, p=0.0001)$.

\section{Discussion}

We have found significant association of the Platelet indices like Platelet count (PLT), platelet distribution width (PDW), MPV, and plateletelcrit (PCT) with psychosocial morbidity like depression, frailty and cognition in elderly subjects. In this study almost all patients with normal (100\%) and high (99.6\%) platelet count had a depression score of more than 5. [ $(\mathrm{p}=0.0001, \mathrm{r}=0.705)$ $\chi 2=811.21, p=0.0031, S]$. In a study by Morel et al. [15] similar results was found as the patients with higher platelet count showed more depression, (they used the Montgomery As berg depression rating scale), while in our study geriatric depression scale were taken [15]. The mean age in Morel et al was $42.94 \pm 9.99$ years. A possible explanation in this context is that depression overlaps with anxiety and panic attack which could lead to sympathetic overdrive; a platelet would respond to adrenalin or noradrenalin released after such stress [15]. In normal platelet count cause of depression is unexplained.
In the group of low platelet count, $50.58 \%$ patients were in robust category according to frailty score, $49.42 \%$ patients were frail and none were disable, having positive correlation between platelet count and frailty score $(r=0.792, p=0.0001)$. Among normal platelet count group, $92.75 \%$ were frail having positive correlation between platelet count and frailty score $(r=0.337, p=0.0001)$. In high platelet count group, $13.40 \%$ were robust, $71.30 \%$ frail and $15.30 \%$ were disable. There was a positive correlation between platelet count and frailty score $(r=0.781, \chi 2=652.91 ; p<0.0001$ S).In contrast to our study, Amira A Mahmoud et al. [16] showed non-significant correlation with platelet count and frailty in the age group of 19-70 years (mean age $48.09 \pm 18$. 3). Phenotypes of frailty used in their study were Cardiovascular health study, and Centre for Epidemiological Studies-Depression. In our study we used FIND questionnaire. In their study $34(24.2 \%)$ patients were robust with mean platelet count of $154.9 \pm 42,22(15.8 \%)$ were prefrail with mean platelet count of $145.7 \pm 71.7$ and $84(60 \%)$ were frail with mean platelet count of $177.4 \pm 70.4$, which was not significant $(\mathrm{p}=0.21)$. In our study patients with low platelet counts, none had MMSE score $\leq 23$, while in high platelet count group $81.01 \%$ had MMSE score $\leq 23,(r=-0.440, \chi 2=754.72, p=0.0001, S)$. No previous study was available for comparison between low platelet count and MMSE score (Table 12).

Table 12: Correlation of mean platelet volume width with MMSE score.

\begin{tabular}{|c|c|c|c|c|c|}
\hline \multirow{2}{*}{ Mean Platelet volume } & \multirow{2}{*}{$\mathbf{n}$} & \multicolumn{2}{|c|}{ MMSE Score } & \multirow{2}{*}{ r-value } & p-value \\
\cline { 2 - 5 } & 290 & $0(0 \%)$ & $290(100 \%)$ & 0.568 & $0.0001, \mathrm{~S}$ \\
\hline $\begin{array}{c}\text { Low(<6.0/cu } \\
\text { micrometer) }\end{array}$ & 456 & $5(1.10 \%)$ & $451(98.90 \%)$ & -0.105 & $0.025, \mathrm{~S}$ \\
\hline $\begin{array}{c}\text { Normal(6-11 cu } \\
\text { micrometer) }\end{array}$ & 254 & $205(80.71 \%)$ & $49(19.29 \%)$ & -0.418 & $0.0001, \mathrm{~S}$ \\
\hline $\begin{array}{c}\text { High(>11 cu } \\
\text { micrometer) }\end{array}$ & 1000 & $210(21 \%)$ & $790(79 \%)$ & -0.388 & $0.0001, \mathrm{~S}$ \\
\hline Total & \multicolumn{2}{|c|}{$0.01(95 \% \mathrm{CI}=0.007-0.045)$} & $731.81, \mathrm{p}=0.0001, \mathrm{~S}$ \\
\hline Odd's Ratio & \multicolumn{2}{|c|}{} \\
\hline
\end{tabular}

When compared with PCT, almost all patients with normal and high PCT had depression score $\geq 5$. Altogether PCT showed positive correlation with depression score $(r=0.831)$ and it was statistically significant $(\mathrm{p}=0.0001)[\chi 2=680.48 ; \mathrm{p}<0.0001]$. No previous studies showed relation of PCT with depression. In our study patients who had low PCT, 131(44.56\%) were robust, 163(55.44\%) were frail and none were disable. Patients with normal PCT almost all $93.42 \%$ were frail and Patients with high PCT, none were robust, $138(52.08 \%)$ were frail and $127(47.92 \%)$ were disable, $(\chi 2=610.95 ; p<0.0001)$. There was no previous study correlating PCT with frailty. In our study there were $79.25 \%$ patients having high PCT had MMSE score $\leq 23,(r=-0.528, \chi 2=737.28, p=0.0001$, S). No separate data for PCT and MMSE score were available. In our study 37/259(14.29\%) patients with low PDW had depression score $\geq 5$, $(r=0.945, p=0.0001)$. While almost all patients with normal and high PDW had depression score $\geq 5,(r=0.743, \chi 2=811.21 ; p<0.0001$ S). No study was available to compare PDW with depression.
In our study patients with low PDW, 131/259 (50.58\%) were robust, $128 / 259$ (49.42\%) were frail and none were disable. There was a positive correlation between PDW and frailty score $(r=0.780)$ and it was statistically significant $(\mathrm{p}=0.0001)$. With normal PDW $447 / 481(92.93 \%)$ patients were frail and 31/481 (6.44\%) were disable, ( $\mathrm{r}=0.283, \mathrm{p}=0.0001)$. In high PDW group, 138/260 (53.08\%) patients were frail and $122 / 260$ (46.92\%) were disable, ( $\chi 2=655.88 ; p<0.0001 \mathrm{~S})$. There was no study of frailty with PDW. In our study when correlated with PDW, 210/260 (80.77\%) patients with high PDW had MMSE score $\leq 23$ and 50/260 (19.23\%) had MMSE score $>23$. Similar correlation was also found by Wang et al. [17] and Liang QC et al. [18] where they got a positive correlation between Mini-Mental State Examination (MMSE) and MPV and PDW, after adjusting confounding factors ( $r=0.532$ for MPV and $r=0.425$ for $P D W, p<0.001$ for both).

In our study patients having low MPV, 68(23.45\%) patients had depression score $\geq 5$ and almost all had depression score $\geq 5$, 
in normal and high MPV group. Out of 1000 patients 223(22.3\%) patients had depression score $<5$ and 777(77.7\%) patients had depression score $\geq 5$, MPV was positively correlated with depression score $(r=0.889)$ and it was statistically significant $(\mathrm{p}=0.002) \quad(\chi 2=693.82 ; \mathrm{p}<0.0001$ S-Significant $)$. Similar results were seen in a study by Ataoglu et al. [19]. In this study out of 1000 patients $134(13.40 \%)$ patients were robust, $712(71.30 \%)$ patients were frail and 153(15.30\%) patients were disable, there was a positive correlation between MPV and frailty score $(r=0.705)$ and it was statistically significant $(p=0.0001)(\chi 2=598.26 ; p<0.0001$ S-Significant). There was no data of frailty with MPV. In our study out of 1000 patients $210(21 \%)$ patients had MMSE score $\leq 23$ and 790(79\%) patients had MMSE score $>23$. $(r=0.388, \chi 2=731.81$; $\mathrm{p}=0.0001 \mathrm{~S}$ ). Similar to our study Wang et al. [17] and Liang QC et al. [18] also compared MPV and PDW with MMSE score, and they showed significant correlation.

\section{Conclusion}

Elderly population are more prone to various psychological, physical and cognitive impairment hence our study provides evidence that platelet indices can be used as a potential biomarker to ascertain probable depression, frailty and cognitive function in elderly population. However more research are required on this topic to generalise in community.

\section{Ethics Committee Approval}

This study was approved by the ethical committee under the terms of the favourable approval by the Data Meghe institute of medical sciences (deemed to be university) [IEC reference numberDMIMS (DU)/IEC/2015-16/1489).

\section{References}

1. Central S Office (2011) Situation analysis of the elderly in India.

2. Yeolekar ME (2005) Elderly in India-needs and issues. J Assoc Physician India 53: 843-844.

3. Kumar V (2012) Geriatric medicine. In: Munjal YP (Ed.), API Textbook of Medicine, ( $9^{\text {th }}$ edn), Jaypee Brothers Medical Publishers (P) Ltd., New Delhi, India, pp. 2038-2042.

4. Ahmed N, Mandel R, Fain MJ (2007) Frailty: an emerging geriatric syndrome. Am J Med 120(9): 748-753.

5. Sunil K (2017) Frailty index-assessment tools in elderly: feasibility in India. Annals of Geriatric Education and Medical Sciences 4(2): 45-49.
6. Leng SX, Hung W, Cappola AR, Yu Q, Xue QL, et al. (2009) White blood cell counts, Insulin like growth factor-1 levels, and frailty in communitydwelling older women. J Gerontol A BiolSci Med Sci 64(4): 499-502.

7. Patel KV, Semba RD, Ferrucci L, Newman AB, Fried LP, et al. (2010) Red cell distribution width and mortality in older adults: a meta-analysis. J Gerontol A BiolSci Med Sci 65(3): 258-265.

8. Endler G, Klimesch A, Sunder-Plassmann H, Schillinger M, Exner M, et al. (2002) Mean platelet volume is an independent risk factor for myocardial infarction but not for coronary artery disease. Br J Haematol 117(2): 399-404.

9. Chu SG, Becker RC, Berger PB, Bhatt DL, Eikelboom JW, et al. (2010) Mean platelet volume as a predictor of cardiovascular risk: a systematic review and meta-analysis. J Thromb Haemost 8(1): 148-156.

10. Leal-Santos FA, Silva SB, Crepaldi NP, Nery AF, Martin TO, et al. (2013) Altered platelet indices as potential markers of severe and complicated malaria caused by Plasmodium vivax: a cross-sectional descriptive study. Malar J 12(1): 462.

11. Pathansali R, Smith NM, Bath PMW (2001) Prothrombotic megakaryocyte and platelet changes in hypertension are reversed following treatment: a pilot study. Platelets 12(3):144-149.

12. Greisenegger S, Endler G, Hsieh K, Tentschert S, Mannhalter C, et al. (2004) Is elevated mean platelet volume associated with a worse outcome in patients with acute ischemic cerebrovascular events? Stroke 35(7): 1688-1691.

13. Parakh K, Sakhuja A, Bhat U, Ziegelstein RC (2008) Platelet function in patients with depression. South Med J 101: 612-617.

14. Markovitz JH, Shuster JL, Chitwood WS, May RS, Tolbert LC (2000) Platelet activation in depression and effects of sertraline treatment: An open-label study. Am J Psychiatry 157(6): 1006-1008.

15. Morel-Kopp MC, Mclean L, Chen Q Tofler GH, Tennant C, et al. (2009) The association of depression with platelet activation: evidence for a treatment effect. J Thromb Haemost 7(4): 573-581.

16. Mahmoud AA, Fouad M, Deraz H (201) Prevalence and Correlates of Frailty among Patients on Maintenance Hemodialysis. Gen Med 3: 156.

17. Wang R, Jin D, Li Y, Liang Q (2013) Decreased mean platelet volume and platelet distribution width are associated with mild cognitive impairment and Alzheimer's disease. J Psychiatr Res 47(5): 644-649.

18. Liang QC, Jin D, Li Y, Wang RT (2014) Mean platelet volume and platelet distribution width in vascular dementia and Alzheimer's disease. Platelets 25(6): 433-438.

19. Ataoglu A, Canan F (2009) Mean platelet volume in patients with major depression: effect of escitalopram treatment. J Clin Psychopharmacol 29(4): 368-371.
Creative Commons Attribution 4.0

International License

For possible submissions Click Here

\section{Submit Article}

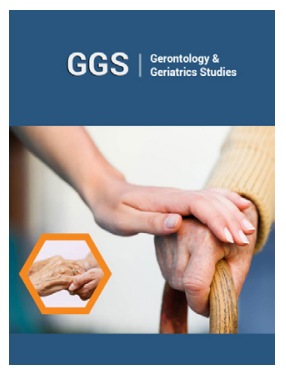

\section{Gerontology \& Geriatrics Studies}

Benefits of Publishing with us

- High-level peer review and editorial services

- Freely accessible online immediately upon publication

- Authors retain the copyright to their work

- Licensing it under a Creative Commons license

- Visibility through different online platforms 